\title{
Monitorização residencial da pressão arterial: atualidades e papel do enfermeiro
}

\author{
HOME BLOOD PRESSURE MONITORING: UPDATES AND THE NURSE'S ROLE
}

\author{
MONITORIZACIÓN DOMICILIARIA DE LA PRESIÓN ARTERIAL: ACTUALIDADES Y \\ PAPEL DEL ENFERMERO
}

\section{Fabiana Agena ${ }^{1}$, Giane Christina Alves da Silva², Angela Maria Geraldo Pierin ${ }^{3}$}

\section{RESUMO}

Trata-se de um artigo de revisão sobre o assunto monitorização residencial da pressão arterial (MRPA) com o objetivo de agregar a contribuição científica atual e apresentar a relevância desta abordagem na assistência ao paciente hipertenso em nosso meio. A técnica oferece vantagens em relação à medida casual, pois proporciona um maior número de medidas, melhor relação com lesão de órgãos-alvo, quantifica o efeito do avental branco, possui boa reprodutibilidade, boa aceitabilidade pelos pacientes, proporciona avaliação da pressão sem a influência do observador e do ambiente do consultório, diminui o número de visitas ao consultório e promove maior adesão ao tratamento. A importância da atuação do profissional enfermeiro na MRPA está ligada ao processo de educação, utilizando estratégias de ensino-aprendizagem, implementando a comunicação equipe-paciente e motivando o paciente a realizar o autocuidado.

\section{DESCRITORES}

Hipertensão.

Determinação da pressão arterial.

Cuidados de enfermagem.

Autocuidado.

\begin{abstract}
This is a review article on home blood pressure monitoring (HBPM) developed with the purpose to increase the current scientific knowledge and present the importance of this approach in the care to patients with hypertension in our setting. This technique has advantages over the causal measurement, as it provides more measurements, a better relationship with the target-organs injuries, it also quantifies the white-coat effect, has good reproducibility, good acceptability by the patients, assesses blood pressure without the influence from the observer and the environment of the appointment, reduces the number of visits to the doctor and promotes greater adherence to treatment. The importance of nursing practice in HBPM is associated with the education process, using teaching-learning strategies, implementing team-patient communication and encouraging patients towards performing self-care.
\end{abstract}

\section{KEY WORDS}

Hypertension.

Blood pressure determination.

Nursing care.

Self care.

\section{RESUMEN}

Se trata de un artículo de revisión sobre el tema de monitorización domiciliaria de la presión arterial (MRPA) con el objetivo de sumar la contribución científica actual y presentar la relevancia de este abordaje en la atención al paciente hipertenso en nuestro medio. La técnica ofrece ventajas en relación a la medida casual, pues proporciona un mayor número de medidas, mejor relación con lesión de órganos-blanco, cuantifica el efecto del delantal blanco, posee buena reproductibilidad, buena aceptación por los pacientes, proporciona evaluación de la presión sin la influencia del observador y del ambiente de consultorio, disminuye el número de visitas al consultorio y promueve mayor adhesión al tratamiento. La importancia de la actuación del personal de enfermería en la MRPA está ligada al proceso de educación, utilizando estrategias de enseñanzaaprendizaje, implementando la comunicación equipo-paciente y motivando al paciente para que realice su autocuidado.

\section{DESCRIPTORES}

Hipertensión.

Determinación de la presión sanguínea.

Atención de enfermería.

Autocuidado.

\footnotetext{
${ }^{1}$ Enfermeira. Mestranda em Ciências da Saúde pela Faculdade de Medicina da Universidade de São Paulo. São Paulo, SP, Brasil. bia.agena@usp.br ${ }^{2}$ Enfermeira. Mestranda em Enfermagem na Saúde do Adulto pela Escola de Enfermagem da Universidade de São Paulo. São Paulo, SP, Brasil. giane.@usp.br ${ }^{3}$ Professora Titular do Departamento de Enfermagem Médico Cirúrgica da Escola de Enfermagem da Universidade de São Paulo. São Paulo, SP, Brasil. pierin@usp.br 


\section{INTRODUÇÃO}

O presente artigo tem como finalidade analisar o papel da Monitorização Residencial da Pressão Arterial (MRPA) no contexto do diagnóstico e tratamento da hipertensão arterial. O interesse por este tema vem crescendo nas últimas décadas, o que pode ser verificado pela crescente produção científica sobre o assunto. Em levantamento realizado na base de dados Medline-Pubmed sobre o tema, sem limitação temporal, com o uso dos descritores home e blood pressure foram localizadas 502 publicações, entre 1958 e 2008. Como pode ser observado na Figura 1, a partir da década de 70 se verifica um incremento na produção científica, quadruplicando na década de 80 e quase triplicando nas décadas seguintes, de 1990 à 2008.

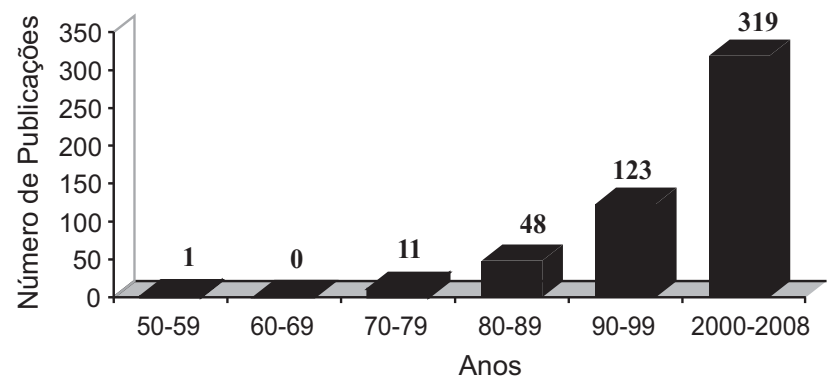

Figura 1 - Evolução do número de publicações científicas sobre Monitorização Residencial da Pressão Arterial, no período de 1950 a 2008.

\section{Hipertensão arterial e a magnitude do problema}

A hipertensão arterial é um grave problema de saúde pública, tendo como desafios a prevenção de lesões de órgãos-alvo e o tratamento mais indicado para cada indivíduo. Constitui o principal fator de risco modificável para doenças cardiovasculares, sendo um fator determinante de causas de mortes prematuras ${ }^{(1-3)}$. Segundo a Organização Mundial de Saúde, atualmente existem 600 milhões de hipertensos no mundo e em seu relatório anual acusa a hipertensão de ser o terceiro principal fator de risco associado à mortalidade mundial, perdendo apenas para sexo inseguro e desnutrição ${ }^{(3)}$.

Dados nacionais indicam prevalências de hipertensão de $23,6 \%$ no estado do Rio Grande do Sul ${ }^{(4)} ; 29,9 \%$ na cidade de Salvador $\mathrm{BA}^{(5)}, 36,4 \%$ na cidade de Goiânia $\mathrm{GO}^{(6)}$; e $22,58 \%$ na cidade de Fortaleza $\mathrm{CE}^{(7)}$. Tais dados confiram que a hipertensão arterial apresenta magnitude expressiva, no contexto nacional.

A hipertensão arterial apresenta controle mundial insatisfatório e a busca de estratégias para o aumento do controle dos níveis pressóricos tem sido cada vez mais abordada na prática clínica ${ }^{(8)}$. O controle adequado da pressão arterial inicia-se com o diagnóstico correto e envolve custos elevados decorrentes principalmente de suas complicações $^{(9)}$.

\section{Medida casual da pressão arterial e a medida realizada fora do consultório}

Pela magnitude da sua importância, o procedimento de medida da pressão arterial deve-se revestir de cuidados visando garantir a acurácia dos valores obtidos. Os membros da equipe de saúde, responsáveis pela realização da medida da pressão arterial, devem prover condições para afastar todas possibilidades de erros que possam comprometer não só o diagnóstico da hipertensão arterial, bem como a condução do tratamento anti-hipertensivo ${ }^{(10)}$. $\mathrm{Na}$ prática clínica ainda existem muitas questões sobre a meIhor forma de avaliar a pressão arterial, seja pela medida casual realizada em visitas a serviços de saúde ou medidas realizadas fora deste ambiente, no domicílio ou ao longo das atividades rotineiras do indivíduo ${ }^{(11)}$.

A medida da pressão arterial pode ser realizada pelo método direto e indireto. O método indireto pode ser realizado de maneira contínua, intermitente e casual, com técnicas auscultatória e oscilométrica ${ }^{(12)}$.

A medida casual da pressão arterial é a mais utilizada para diagnóstico e tratamento da hipertensão arterial, realizada pelos profissionais de saúde com o uso de esfignomanômetros de coluna de mercúrio, aneróide ou aparelhos automáticos, em ambiente de saúde, seja na atenção básica, consultórios ou outros locais institucionalizados.

Observa-se, em diversos estudos, que a medida da pressão arterial realizada em consultório apresenta valores mais elevados do que a registrada por Monitorização Ambulatorial de Pressão Arterial (MAPA), que permite avaliação dos níveis tensionais por $24 \mathrm{~h}$ enquanto o indivíduo realiza suas atividades habituais, no período de vigília e sono e pela medida residencial. Tal fato relaciona-se ao fenômeno do avental branco, representado pela hipertensão do avental branco e efeito do avental branco. A hipertensão do avental branco ocorre quando o nível de pressão arterial está acima de 140/90 mmHg quando medido em consulta, mas apresenta níveis normais quando avaliada pela MAPA e/ou MRPA ${ }^{(13)}$. O efeito do avental branco também tem sido relacionado com a resposta da pressão arterial na presença do médico e é caracterizado por níveis mais elevados de pressão arterial em consultório, independente do diagnóstico de hipertensão ou normotensão(14). Em estudo realizado em Unidades Básicas de Saúde comparando a média dos quatro dias de medida residencial e a medida realizada pela enfermeira na unidade mostrou que não houve diferença para a pressão sistólica, porém a pressão diastólica residencial foi significativamente menor que a registrada pela enfermeira caracterizando o efeito do avental branco ${ }^{(15)}$.

\section{Monitorização Residencial da Pressão Arterial}

A medida realizada no domicílio passou a ser estudada com maior propriedade após o desenvolvimento de apareIhos automáticos, que independem da habilidade do indivíduo de medir a própria pressão arterial, com possibilidade de grande número de medidas em situações fora do con- 
sultório médico. A MRPA é o registro sistematizado da pressão arterial, pela manhã e à noite, durante cinco dias, realizado pelo paciente ou outra pessoa treinada, durante a vigília, no domicílio ou no trabalho(16). A MRPA deve ser diferenciada de auto registro, realizado de forma esporádica pelo hipertenso.

Estudos apontam que a MRPA é um método alternativo melhor do que a medida de consultório e tão boa quanto a monitorização ambulatorial da pressão arterial na avaliação de risco cardiovascular da população geral e de hipertensos ${ }^{(17-18)}$. Investigação realizada em um ambulatório de um Hospital de Ensino na cidade de São Paulo, evidenciou o efeito benéfico da MRPA na avaliação do controle dos hipertensos, superando inclusive a avaliação da MAPA. O estudo destaca ainda, que a pressão revelada pela MRPA foi menor que a obtida pela MAPA e ambas menores que a obtida no consultório, o que confirma achados da literatura de que a pressão arterial de consultório é mais elevada do que as obtidas nos métodos $\operatorname{citados}^{(19)}$.

\section{Indicações e vantagens da MRPA}

A utilização da MRPA na confirmação do diagnóstico de hipertensão arterial é indicada em diretrizes voltadas para o manejo da hipertensão arterial como método alternativo, podendo ser utilizada sempre que disponível, associada à medida de consultório e MAPA ${ }^{(20)}$. Para a Sociedade Européia de Hipertensão, a MRPA é mais apropriada que a medida casual para o acompanhamento de pacientes devido ao baixo custo e a conveniência para a repetição das medidas. Promove também, informação suplementar permitindo um diagnóstico preciso e melhor acompanhamento da hipertensão ao longo do tratamento ${ }^{(21)}$. As inúmeras vantagens da MRPA podem ser observadas no Quadro 1.

Quadro 1 - Vantagens da monitorização residencial da pressão arterial em relação à medida casual ${ }^{(16)}$.

\begin{tabular}{|l|}
\hline $\begin{array}{l}\text { Apresenta um maior número de medidas durante o dia e por vários } \\
\text { dias, semanas ou meses }\end{array}$ \\
\hline Avaliação dos efeitos do tratamento em diferentes momentos do dia \\
\hline Não há reação de alarme \\
\hline Boa aceitabilidade pelos pacientes \\
\hline Boa reprodutibilidade e valor prognóstico \\
\hline $\begin{array}{l}\text { Proporciona avaliação da pressão sem a influência do observador e } \\
\text { do ambiente do consultório }\end{array}$ \\
\hline Melhor relação com lesão de órgão salvo \\
\hline Diminui o número de visitas ao consultório \\
\hline Atenua os erros e preferências do observador \\
\hline $\begin{array}{l}\text { Possibilita o armazenamento, impressão e transmissão a distância } \\
\text { das leituras obtidas }\end{array}$ \\
\hline Quantifica o efeito do avental branco \\
\hline $\begin{array}{l}\text { Promove maior adesão ao tratamento e envolvimento no manejo } \\
\text { da hipertensão }\end{array}$ \\
\hline
\end{tabular}

\section{Protocolo para realização da MRPA}

A II Diretriz para Uso da Monitorização Residencial da Pressão arterial sugere a realização durante cinco dias úteis da semana, sendo o primeiro dia reservado para instruções, treinamento e seleção do braço com valores mais elevados de pressão arterial no qual serão realizadas as medidas. Nos quatro dias seguintes, a pressão arterial deve ser medida pelo menos três vezes pela manhã e três vezes à noite, no período compreendido entre $06-10 \mathrm{~h}$ e 18-22 h. O resultado da MRPA é considerado anormal quando a média de pressão sistólica estiver acima de 135 e/ou diastólica acima de $85 \mathrm{mmHg}^{(16)}$.

Para interpretação dos dados, no relatório de MRPA deverá constar as médias diárias e total, e para o registro ser aceito como válido deverá atingir pelo menos 12 medidas válidas. As médias serão obtidas com registros efetivos no mínimo de quatro dias, desprezando-se os valores obtidos no primeiro dia da monitorização, porém estes valores deverão constar no laudo para a avaliação da reação de alarme. Deverão ser excluídas medidas aberrantes desde que não haja justificativa clínica ${ }^{(16,22)}$.

Para realização do procedimento de aferição da pressão arterial o indivíduo deve ser orientado conforme mostra o Quadro 2.

Quadro 2 - Orientações ao paciente para a realização da MRPA(16).

\begin{tabular}{|l|}
\hline Realizar as medidas em ambiente tranqüilo com temperatura agradável \\
\hline Estar de bexiga vazia \\
\hline Não ter praticado exercício físico há $60-90$ minutos \\
\hline $\begin{array}{l}\text { Não ingerir bebidas alcoólicas, café, alimentos ou tabaco } 30 \text { minutos } \\
\text { antes }\end{array}$ \\
\hline Não falar durante a medida \\
\hline $\begin{array}{l}\text { Efetuar as medidas antes da ingestão dos medicamentos e antes do } \\
\text { desjejum e do jantar, ou após duas horas }\end{array}$ \\
\hline $\begin{array}{l}\text { Realizar a medida em posição sentada após } 2 \text { minutos de repouso, com } \\
\text { as costas apoiadas e pernas descruzadas, com intervalo entre as } \\
\text { medidas de } 1 \text { minuto }\end{array}$ \\
\hline $\begin{array}{l}\text { Colocar o manguito no braço livre de roupas e sem garroteamento por } \\
\text { roupas apertadas }\end{array}$ \\
\hline $\begin{array}{l}\text { Utilizar sempre o mesmo braço definido nas instruções, apoiado na } \\
\text { altura do coração, com a palma da mão voltada para cima e sem } \\
\text { movimentação durante as medidas }\end{array}$ \\
\hline
\end{tabular}

\section{Aparelhos de MRPA}

Para a monitorização confiável na prática clínica, são recomendados aparelhos automáticos ou semi-automáticos com deflagração manual, para realização de medida no braço, porém os aparelhos automáticos são mais indicados pela facilidade do manuseio. Todos aparelhos devem ser validados de acordo com as normas internacionais, como da British Hypertension Society (BHS) e a Association for the Advancement of Medical Instrumentation (AAMI)(23). Aparelhos que não atenderem ao critério da AAMI ou apresentarem classificação C ou D pelo BHS para a pressão sistólica ou diastólica não são recomendáveis ${ }^{(24)}$. 
$\mathrm{Na}$ aquisição do aparelho alguns fatores deverão ser considerados, além da validação, tais como o custo do aparelho e do software, memória suficiente para realização do protocolo, possibilidade de impressão dos dados, manual contendo instruções de forma adequada, custo de manutenção, custo do material de consumo, manguitos de diversos tamanhos $^{(25)}$, assistência técnica disponível e tempo de garantia ${ }^{(16)}$. Um recurso que pode ser usado para avaliar a confiabilidade de um aparelho automático de medida da pressão arterial é consultar a listagem de aparelhos validados segundo os protocolos internacionais, publicada no site da British Hypertension Society ${ }^{(23)}$. Infelizmente, a grande maioria destes aparelhos não está disponível no mercado nacional, e aqueles disponíveis, possuem preço elevado o que dificulta a utilização mais ampla, principalmente em serviços públicos.

\section{Papel do enfermeiro}

O papel do enfermeiro na hipertensão arterial, doença multifatorial que requer uma abordagem multiprofissional, tem como objetivo a promoção da saúde. A consulta de enfermagem junto aos hipertensos é uma estratégia que propicia grandes benefícios. A educação sobre doença e a orientação sobre hábitos de vida saudáveis, com foco em mudanças de atitude frente ao estilo de vida de forma clara, tem como objetivo um maior esclarecimento sobre a patologia, promoção do autocuidado e conseqüentemente melhor controle pressórico e adesão a terapêutica proposta. Na MRPA o enfermeiro busca a excelência no atendimento com o objetivo de proporcionar um treinamento de forma clara, onde o paciente sinta-se à vontade para esclarecer suas dúvidas e realize o protocolo de exame da melhor forma possível. $\mathrm{O}$ enfermeiro se faz presente em todas as etapas deste processo que se inicia a partir da escolha do aparelho e perdura durante a orientação do manejo deste, do preenchimento do diário de atividades, da realização do exame propriamente dito e finaliza na emissão do relatório. A satisfação do paciente durante este processo tem relação direta com a maneira de realização do protocolo e uma das formas de assegurar o conforto e segurança durante este processo é a disponibilidade de um contato direto, portanto é aconselhável prover um número de telefone para que o paciente possa esclarecer dúvidas, durante a realização da MRPA.

\section{O uso da MRPA na pesquisa clínica}

Estudos mostram que a MRPA melhora a adesão à terapêutica anti-hipertensiva, propiciando aumento do número de hipertensos que alcançam a meta terapêutica, mesmo em níveis de atenção primária à saúde, melhorando os índices de controle de pressão $\operatorname{arterial}^{(16,26)}$.

Outras investigações têm evidenciado a relevância da MRPA no manejo dos hipertensos. O estudo PAMELA (Pressione Arteriose Monitorate E Loro Associazioni) acompanhou prospectivamente mais de 2000 pacientes representantes da população geral de uma região do norte da Itália, por um período médio de 131 meses, utilizando pressão arterial de consultório, MAPA e MRPA. Independente da forma com que foi medida a pressão arterial, houve relação direta e exponencial entre os valores iniciais de pressão arterial e mortalidade cardiovascular ${ }^{(27)}$. O estudo SAMPLE (Study on Ambulatory Monitoring of Pressure and Lisinopril Evaluation), monitorou a evolução de hipertrofia ventricular esquerda em pacientes sob uso de anti-hipertensivo e revelou no final que a MAPA e a MRPA tiveram melhor poder prognóstico do que a medida casual de consultório ${ }^{(28)}$.

Em estudo onde se correlacionou os valores da pressão sistólica, diastólica e a pressão de pulso com o índice de massa do ventrículo esquerdo com uso da MRPA, o principal achado mostrou a presença de correlação positiva entre o índice de massa de ventrículo esquerdo e a pressão de pulso, concluindo que o índice de massa do ventrículo esquerdo aumenta concomitantemente com os incrementos da pressão arterial sistólica e na pressão de pulso, e que essa correlação é mais significativa na pressão de pulso ${ }^{(29)}$. Publicação recente realizada no Japão a partir da simulação de gastos para implementação da MRPA usando dados de estudo clínico realizado na cidade de Ohasama, constatou que quando a MRPA não é incorporada ao processo diagnóstico, o custo médico está estimado em US\$ 10,89milhões/1000pacientes/5anos. Porém, quando a MRPA está incorporada, os gastos caem para US\$ 9,33miIhões/1000pacientes/5anos, o que representa uma economia que varia entre US\$ 674 mil à US\$251milhões/1000pacientes/5anos no tratamento da hipertensão. Os autores do estudo concluem que a introdução da MRPA é muito útil para a redução dos custos com saúde ${ }^{(30)}$.

Os estudos HOMERUS (Home versus Office blood pressure MEasurements: Reduction of Unnecessary treatment Study) e THOP Trial (Treatment of Hypertension According to Home or Office Blood Pressure) verificaram que o tratamento medicamentoso com a introdução da MRPA trouxe benefício ao paciente quando comparado ao tratamento convencional. Ao final dos estudos um percentual maior de pacientes acompanhados com MRPA necessitou de tratamento medicamentoso menos intensivo, o que comprovou uma diminuição dos custos $^{(31-32)}$. Em nosso meio, em estudo que comparou a MRPA e a MAPA com os registros de consultório e correlacionouo índice de massa de ventrículo esquerdo com a MRPA e medida de consultório, a MRPA teve melhor correlação com lesão de órgãos-alvo do que a medida da pressão de consultório(33). Em outra investigação que comparou a medida de pressão arterial realizada em casa por médicos, enfermeiras e pacientes com a medida de pressão arterial de consultório, MAPA e MRPA, mostrou que a medida que mais se aproximou com a MRPA e a MAPA foi a medida realizada pelo paciente e a MRPA e a MAPA apresentaram bom valor prognóstico em relação às medidas realizada em consultório ${ }^{(34)}$.

\section{CONCLUSÃO}

O presente artigo destacou a importância no manejo dos hipertensos através da monitorização residencial da 
pressão arterial. A MRPA é um método que permite a avaliação do comportamento da pressão arterial a médio e longo prazo, sendo eficaz na avaliação da terapêutica antihipertensiva, podendo ter vantagem em relação à MAPA por ser um método com maior aceitabilidade entre os indivíduos e mais barato.

A revisão mostrou um consenso com a indicação do método em bibliografia atual, ressaltando a grande aceitação no meio científico. Apesar de ser uma técnica difundida mundialmente ainda há discussões quanto ao número de aferições e período a ser realizada. Revisão sobre o assun-

\section{REFERÊNCIAS}

1. Chobanian AV, Bakris GL, Black HR, Cushman WC, Green LA, Izzo JL Jr, et al. The Seventh Report of the Joint National Committee on Prevention, Detection, Evaluation, and Treatment of High Blood Pressure: the JNC 7 report. JAMA. 2003;289(19):2560-72.

2. Ezzati M, Lopez AD, Rodgers A, Vander Hoorn S, Murray CJ. Selected major risk factors and global and regional burden of disease. Lancet. 2002;360(9343):1347-60.

3. World Health Organization. The World Health Report 2003 [Internet]. Geneva; 2003 [cited 2008 Oct 20]. Available from: http://www.who.int/whr/2003/en/whr03_en.pdf

4. Costa JSD, Barcellos FC, Sclowitz ML, Sclowitz IKT, Castanheira $M$, Olinto MTA, et al. Prevalência de hipertensão arterial em adultos e fatores associados: um estudo de base populacional urbana em Pelotas, Rio Grande do Sul, Brasil. Arq Bras Cardiol. 2007; 88(1):59-65.

5. Lessa I, Magalhães L, Araújo MJ, Almeida Filho N, Aquino E, Oliveira MMC. Hipertensão arterial na população adulta de Salvador (BA) - Brasil. Arq Bras Cardiol. 2006;87(6):747-56.

6. Jardim PCBV, Gondim MRP, Monego ET, Moreira HG, Vitorino PVO, Souza WKSB, et al. Hipertensão arterial e alguns fatores de risco em uma capital brasileira. Arq Bras Cardiol. 2007;88(4):452-7.

7. Feijão AMM, Gadelha FV, Bezerra AA, Oliveira AM, Silva MSS, Lima JWO. Prevalência de excesso de peso e hipertensão arterial, em população urbana de baixa renda. Arq Bras Cardiol. 2005;84(1):29-36.

8. Colósimo FCC, Silva SSBE, Pierin AMG. Medida residencial da pressão arterial (MRPA) e programa de educação para hipertensos: ferramentas de incremento do controle. Hipertensão. 2008;11(2):55-60.

9. MacMahon S, Rodgers A, Neal B, Chalmers J. Blood pressure lowering for the secondary prevention of myocardial infarction and stroke. Hypertension. 1997;29(2):537-8. to ${ }^{(22)}$ mantém a recomendação de avaliação da pressão durante sete dias, com descarte do primeiro para análise, mesmo face a estudos que realizaram as medidas em um número menor de dias. Outro ponto relevante sobre o método é a possibilidade de aquisição de medidas de pressão arterial sem a interferência do examinador e do ambiente, que continua sendo o maior benefício desta técnica na prática clínica. Apesar da MRPA oferecer vantagens, a medida convencional de consultório para diagnóstico e controle da pressão arterial continua sendo o método padrão utilizado em nosso meio, enquanto que a MRPA aparece como método complementar de avaliação da pressão arterial.

10. Rabello CCP, Pierin AMG, Mion Junior D. O conhecimento insatisfatório de profissionais da área de saúde sobre a medida da pressão arterial. Rev Esc Enferm USP. 2004;38(2):127-34.

11. Sociedade Brasileira de Cardiologia; Sociedade Brasileira de Hipertensão; Sociedade Brasileira de Nefrologia. V Brazilian Guidelines in Arterial Hypertension. Arq Bras Cardiol. 2007;89(3):e24-79.

12. Pierin AMG. Uma proposta para o cuidar. Barueri: Manole; 2004. p. 27-48.

13. Segre CA, Ueno RK, Warde KRJ, Accorsi TAD, Miname MH, Chi $\mathrm{CK}$, et al. White-coat hypertension and normotension in the league of hypertension of the Hospital das Clínicas, FMUSP. Prevalence, clinical and demographic characteristics. Arq Bras Cardiol. 2003; 80(2):117-21.

14. Myers GM, Reeves RA. White coat effect in treated hypertensive patients: sex differences. J Hum Hypertens. 1995;9(9):729-33.

15. Pierin AMG, Alavarce DC, Santos GAS. Medida residencial da pressão arterial em hipertensos atendidos em Unidades Básicas de Saúde. Hipertensão. 2006;9(2):60-4.

16. Alessi A, Brandão AA, Pierin A, Feitosa AM, Machado CA, Moraes Forjaz $\mathrm{CL}$, et al. IV Guideline for ambulatory blood pressure monitoring. II Guideline for home blood pressure monitoring. IV ABPM/II HBPM. Arq Bras Cardiol. 2005;85 Suppl 2:1-18.

17. Ohkubo T, Imai Y, Tsuji I, Nagai K, Kato J, Kikuchi N, et al. Home blood pressure measurement has a stronger predictive power for mortality than does screening blood pressure measurement: a population-based observation in Ohasama, Japan. J Hypertens. 1998;16(7):971-5.

18. Bobrie G, Chatellier G, Genes N, Clerson P, Vaur L, Vaisse B, et al. Cardiovascular prognosis of "masked hypertension" detected by blood pressure self-measurement in elderly treated hypertensive patients. JAMA. 2004;291(11):1342-9. 
19. Pierin AMG, Costa KRA, Gusmão JL, Caetano El, Ortega K, Mion Junior $\mathrm{D}$. $\mathrm{O}$ efeito benéfico da medida residencial da pressão arterial (MRPA) na avaliação do controle da hipertensão arterial. Hipertensão. 2007;10(2):62-5.

20. Myers MG, Tobe SW, McKay DW, Bolli P, Hemmelgarn BR, McAlister FA. New algorithm for the diagnosis of hypertension. Am J Hypertens. 2005;18(10):1369-74.

21. Stergiou GS, Mengden T, Padfield PL, Parati G, O’Brien E. Self monitoring of blood pressure at home. BMJ. 2004;329(7471):870-1.

22. Parati G, Stergiou GS, Asmar R, Bilo G, de Leeuw P, Imai Y, et al. European Society of Hypertension guidelines for blood pressure monitoring at home: a summary report of second international consensus conference on home blood pressure monitoring. J Hypertens. 2008; 26(8):1505-30.

23. British Hypertension Society [Internet]. [cited 2008 Nov 11]. Available from: http://www.bhsoc.org/default.stm

24. Mano GMP, Souza VF, Pierin AMG, Lima JC, Ignes EC, Ortega KC, et al. Assessment of the DIXTAL DX-2710 automated oscillometric device for blood pressure mensurement with the validation protocols of the british hypertension society (BHS) and the association for the advancement of medical instrumentation (AAMI). Arq Bras Cardiol. 2002;79(6):606-10.

25. Pierin AMG. A relação entre o tamanho do manguito e o braço na medida indireta da pressão arterial. Hipertensão. 2005;8(3):107-8.

26. Marquez-Contreras E, Martell-Claros N, Gil-Guillen V, de la Figuera-Von WM, Casado-Martinez JJ, Martin-de Pablos JL, et al. Efficacy of a home blood pressure monitoring programme on therapeutic compliance in hypertension: the EAPACUM-HTA study. J Hypertens. 2006;24(1):169-75.

27. Sega R, Facchetti R, Bombelli M, Cesana G, Corrao G, Grassi G, et al. Prognostic value of ambulatory and home blood pressures compared with office blood pressure in the general population: follow-up results from the Pressioni Arteriose Monitorate e Loro Associazioni (PAMELA) study. Circulation. 2005;111(14):1777-83.
28. Mancia G, Zanchetti A, Agabiti-Rosei E, Benemio G, De Cesaris $R$, Fogari $R$, et al. Ambulatory blood pressure is superior to clinic blood pressure in predicting treatment-induced regression of left ventricular hypertrophy. SAMPLE Study Group. Study on Ambulatory Monitoring of Blood Pressure and Lisinopril Evaluation. Circulation. 1997;95(6):1464-70.

29. De Marco A, Feitosa AM, Gomes MM, Parente GB, Victor EG. Pressão de pulso obtida por Monitorização Residencial da Pressão Arterial e sua relação com o índice de Massa do Ventrículo Esquerdo. Arq Bras Cardiol. 2007;88(1):91-5.

30. Fukunaga H, Ohkubo T, Kobayashi M, Tamaki Y, Kikuya K, Metoki $\mathrm{H}$, et al. Cost-effectiveness of the introduction of home blood pressure measurement in patients with office hypertension. J Hypertens. 2008;26(4):685-90.

31. Verberk WJ, Kroon AA, Lenders JWM, Kessels AGH, Montfrans GA, Smit AJ, et al. Self-measurement of blood pressure at home reduces de need for antihypertensive drugs: a randomized, controlled trial. Hypertension. 2007;50(6):101925.

32. Staessen JA, Den Hond E, Celis H, Fagard R, Keary L, Vandenhoven $\mathrm{G}$, et al. Antihypertensive treatment based on blood pressure measurement at home or in the physician's office: a randomized controlled trial. JAMA. 2004;25;291(8):955-64.

33. Gomes MA, Pierin AMG, Segre CA, Mion Junior D. Monitorização residencial da pressão arterial e monitorização ambulatorial da pressão arterial versus medida da pressão arterial no consultório. Arq Bras Cardiol. 1998;71(4):66-78.

34. Pierin AMG, Ignez EC, Jacob Filho W, Barbato AJG, Mion Junior D. Blood pressure measurements taken by patients are similar to home and ambulatory blood pressure measurements. Clinics. 2008;63(1):43-50. 